Research Paper

\title{
Influence of Endodontic Treatment on Systemic Oxidative Stress
}

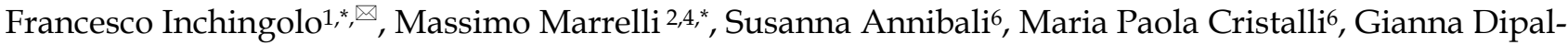 \\ $\mathrm{ma}^{2}$, Alessio Danilo Inchingolo ${ }^{7}$, Antonio Palladino7, Angelo Michele Inchingolo ${ }^{5}$, Marco Gargari ${ }^{8}{ }^{*}$, Marco \\ Tatullo $2,3,4,{ }^{*}$
}

1. Department of interdisciplinary Medicine, University of Bari, General Hospital, Bari, Italy;

2. Unit of Maxillofacial Surgery, Calabrodental, Crotone, Italy;

3. Department of Basic Medical Sciences, University of Bari, Bari, Italy;

4. Tecnologica Research Institute, Biomedical Section, Crotone, Italy;

5. Department of Surgical, Reconstructive, and Diagnostic Sciences, University of Milan, Milan, Italy;

6. Department of Stomatology and Maxillofacial Science - Oral Surgery Unit - University of Rome "Sapienza" Rome, Italy;

7. Medical School, University of Bari, Bari, Italy;

8. Department of Clinical Sciences and Translational Medicine, University of Rome "Tor Vergata", Rome, Italy.

* These Authors equally contributed to this article.

Corresponding author: Prof. Francesco Inchingolo MD, DMD. Dept. of Interdisciplinary Medicine (DIM), University of Bari, Pl.ce G. Cesare, 70124 Bari - Italy.

() Ivyspring International Publisher. This is an open-access article distributed under the terms of the Creative Commons License (http://creativecommons.org/ licenses/by-nc-nd/3.0/). Reproduction is permitted for personal, noncommercial use, provided that the article is in whole, unmodified, and properly cited.

Received: 2013.05.09; Accepted: 2013.08.26; Published: 2013.12.06

\begin{abstract}
Introduction: An increased production of oxidizing species related to reactive oral diseases, such as chronic apical periodontitis, could have systemic implications such as an increase in cardiovascular morbidity.

Based on this consideration, we conducted a prospective study to assess whether subjects affected by chronic periodontitis presented with higher values of oxidative stress than reference values before endodontic treatment, and whether endodontic treatment can reduce the oxidative imbalance and bring it back to normal in these subjects.

Materials and methods: The authors recruited 2 groups of patients from private studies and dental clinics: these patients were recruited randomly. The oxidative balance in both patients with chronic apical periodontitis (CAP) and healthy control patients was determined by measuring the oxidant status, using an identification of the reactive oxygen metabolites (d-ROMs) test, while the antioxidant status in these patients was determined using a biological antioxidant potential (BAP) test. Both these tests were carried on plasma samples taken from enrolled patients. Values were measured both before the endodontic treatment of the patients with chronic apical periodontitis, and 30 and 90 days after treatment, and compared to those obtained from healthy control patients.

Results: It was found that, on recruitment, the patients with chronic apical periodontitis exhibited significantly higher levels of oxidative stress than control patients, as determined by the d-ROMs and BAP tests. Furthermore, the d-ROMs test values were shown to decrease and the BAP test values to increase over time in patients with chronic apical periodontitis following endodontic therapy. As the levels of oxidative stress in these patients tended to reduce and return to normal by 90 days following treatment.

Conclusions: This study has demonstrated a positive association between chronic apical periodontitis and oxidative stress. Subjects affected by chronic apical periodontitis are exposed to a condition of oxidative stress, which is extremely dangerous to general health. Moreover, one can infer from these findings that through proper endodontic therapy, a good oxidative balance can be restored, thereby avoiding the risk of contracting the abovementioned diseases.
\end{abstract}

Key words: Oxidative stress; chronic apical periodontitis; endodontic treatment. 


\section{Introduction}

Reactive chemical species (RCS) are highly reactive molecules, atoms, or ions that are the intermediates of physiological reactions involved in controlling important vital processes. At the structural level, RCS can be distinguished between reactive oxygen species (ROS), such as superoxide anion, hydroxyl radical, singlet oxygen, and hydrogen peroxide; reactive nitrogen species (RNS); reactive carbonyl species, and reactive chlorine species. Once they are produced, ROS are able to trigger propagation reactions in which radicals react with other organic molecules and cause addition, rearrangement, fragmentation, or transfer reactions ${ }^{1}$ that can result in oxidative damage. Since ROS are potentially harmful, the body has developed antioxidant defense mechanisms that include several enzymatic and non-enzymatic systems. These include the peroxidase system; proteins such as lactoferrin, ferritin, superoxide dismutases, albumin, and ceruloplasmin; vitamins A, E, and C; ubiquinone; lipoic acid; flavonoids; thiols; and uric acid ${ }^{2}$. Antioxidant defense systems, along with other mechanisms such as inactivation, repair, and removal, act to reduce oxidative stress and protect against oxidative damage.

Increases in ROS concentration can be observed not only in response to physiological conditions, but also as a result of infections and pathological conditions. The oxidative damage that can result from higher ROS concentrations can affect any physiological structural element, including carbohydrates, lipids, and amino acids; however, it is unclear to what extent ROS are the cause or effect of the disease under consideration. Indeed, the relation between pathological or physiopathological conditions and oxidative stress is difficult to diagnose, because the latter does not produce specific clinical features ${ }^{3,4}$. Despite this, an association was first noticed in the 1950s, when the occurrence of a severe form of cataract that causes blindness, Retrolental Fibroplasia, was found to be correlated with the administration of oxygen at higher partial pressures than those existing in the atmosphere immediately following birth. This hyperbaric therapy was subsequently proven to impact the production of ROS, resulting in the clouding of the crystalline lens in newborns ${ }^{3,4}$. Oxidative stress has since been correlated with several pathological or physiopathological conditions, including type 2 diabetes mellitus 3,4, senescence 5, 6, cardiovascular disease 7-11, cancer 12-14, pulmonary disease 15, 16, and hepatopathies ${ }^{15,16}$. However, to date, no study has investigated the link between oxidative stress and diseases of endodontic origin.

As there is an exact balance between the production and elimination of ROS in the oral cavity, an increase in the production and/or a reduction in the elimination of ROS could increase the risk of oral diseases. Furthermore, an increased production of oxidizing species related to reactive oral diseases such as chronic apical periodontitis could have systemic implications such as an increase in cardiovascular morbidity. Indeed, many bacterial species may participate in the pathogenesis of endodontic diseases: in addition to the bacteria that are usually detected in chronic apical periodontitis, frequently, radicular dentin of teeth with periodontitis can be also invaded by putative periodontal pathogens, such as Prevotella intermedia, Phorphyromonas gingivalis, Fusobacterium nucleatum, Bacteroides forsythus, Peptostreptococcus micros, and Streptococcus intermedius ${ }^{17}$. The increase of inflammatory processes mediated by these pathogens, together with the recruitment of leukocytes by chemotaxis, create an oxidative attack directly dependent on the intensity of the infection ${ }^{18}$.

The ROS are undoubtedly one of the most effective pathogenic mechanisms in all those bacterial pathologies which lead to bone resorption ${ }^{19}$ and several scientifical evidences show how the oxidative killing, carried out from neutrophils against pathogenic bacteria, is frequently the first stage of bone damage; especially in some dental pathologies, such as the acute apical periodontitis (AAP), the neutrophils obtained from peripheral blood of AAP subjects shown increased production of hydrogen peroxide and superoxide anion, which is instead normalized after surgical treatment ${ }^{20}$.

Based on these considerations, we have conducted a prospective study to assess whether subjects affected by chronic periodontitis presented with higher values of oxidative stress than reference values before endodontic treatment, and whether endodontic treatment can reduce the oxidative balance and bring it back to normal in these subjects. Our study is the first to be carried out on plasma samples, in order to assess the potential systemic implications arising from the oxidative stress condition which might result from an untreated endodontic pathology.

Some studies have already addressed the correlation between inflammatory conditions, acute or chronic, which may affect the periodontium or the dental pulp, and the related changes in biomarkers found in crevicular fluid; moreover, some studies have clearly shown that crevicular transudate does not have a cellular, proteic and enzymatic component comparable to that which is identifiable in circulating plasma.

It's clear that our study, analyzing the hypothetical correlation between a chronic apical periodontitis and an imbalance of the systemic oxidative stress, it leads to reconsider the endodontic pathology with a different attention. 


\section{Materials and Methods}

\section{Study Participants}

The authors recruited 2 groups of patients from private studies and dental clinics: these patients were recruited randomly, were informed regarding the study and they have signed an informed consent. The study was conducted in compliance with the "Ethical principles for medical research involving human subjects" of Helsinki Declaration. The University Research Ethics Committee (U.R.E.C.) has revised and finally approved this study. The study was conducted in accordance with Italian laws and regulations.

"Group 1" was formed by 33 patients (21 men and 12 women) between 30 and 68 years of age with a diagnosis of chronic apical periodontitis (CAP) of only one tooth; the diagnosis was carried out in accordance with what has been described previously by Gutmann ${ }^{21}$. Exclusion criteria: the authors excluded from this study all patients subjected to any potential treatment or condition that could alter the results, such as anti-inflammatory or antibiotic administration in the 3 months before recruitment, smoking habit, obesity, systemic inflammatory of metabolic diseases and poor oral hygiene. The teeth affected with CAP were all bi/multi rooted. All of these patients presented with a single lesion with a radiographic diameter of $2 \mathrm{~cm}$ or less that did not affect anatomical structures such as the maxillary sinus or the inferior alveolar nerve. "Group 2" included 103 patients (58 men and 45 women) between 30 and 68 years of age who had no signs of chronic apical periodontitis 21 and were healthy. Patients were excluded from this study if there was no healing of chronic periodontitis. Informed consent was received from all study participants. Parameters for healing of CAP were defined in accordance with what has been described previously by Gutmann et al. (2009) ${ }^{21}$.

\section{Assessment of Oxidative Stress}

Oxidative stress assessment was performed by means of an integrated analytical system composed of a photometer and a mini-centrifuge (FRAS4, H\&D s.r.l., Parma, Italy). Samples of whole capillary blood were centrifuged, immediately after being harvested by finger puncture, at $6000 \times \mathrm{g}$ for $5 \mathrm{~min}$ and $10 \mu \mathrm{L}$ of plasma tested for total oxidant capacity (using a d-ROMs test kit) and BAP as iron-reducing activity (using a BAP test kit) (Diacron International s.r.l., Grosseto, Italy). The d-ROMs test is based on the ability of a plasma sample to oxidize the $\mathrm{N}, \mathrm{N}$-diethylparaphenilendiamine (colorless) to its radical cation (red); the reaction is monitored photometrically at $37^{\circ} \mathrm{C}$ at $505 \mathrm{~nm}$, and the results are expressed as Carratelli Units (CARR U, $\triangle$ Abs5050 $\mathrm{nm} / \mathrm{min})$. The normal values of a d-ROMs test range between 250 and 300 CARR U. The BAP test is based on the ability of a plasma sample to reduce the iron of a colored complex containing ferric ions to its colorless ferrous derivative; the reaction is monitored photometrically at $37^{\circ} \mathrm{C}$ at $505 \mathrm{~nm}$, and the results are expressed in $\mu \mathrm{mol} / \mathrm{L}$ of reduced iron using ascorbic acid as a standard. The normal value of a BAP test is $>2200 \mu \mathrm{M}$. To maintain consistency, the same set of kits was used for all tests, and all tests were carried out by the same operator using the same machine. The analytical instrumentation was also calibrated before the analytical seat with serum standards with known $\mathrm{d}-\mathrm{ROMs}$ and BAP values.

\section{Timeline}

The diagnosis and treatment plan was established for "Group 1" patients at Time $\left(\mathrm{T}_{0}\right)$. A sample of capillary blood was also taken from each participant at $\mathrm{T}_{0}$, including the control patients recruited in "Group 2"; determination of reactive oxygen metabolites (d-ROMs) tests to assess the oxidant status and biological antioxidant potential (BAP) tests to assess the antioxidant status in these patients were performed in both the groups. The endodontic treatment of patients in "Group 1" was performed conventionally 7 days later $\left(T_{1}\right)$. All "Group 1" patients were then called back for clinical and radiographic evaluation 30 days after the completion of the endodontic therapy $\left(\mathrm{T}_{2}\right)$. BAP tests and d-ROMs tests on samples taken from both these groups. Finally, a clinical and radiographic evaluation of the endodontic lesions of patients recruited in "Group 1" was carried out conventionally 60 days after $\mathrm{T}_{2}\left(\mathrm{~T}_{3}\right)$, and BAP tests and d-ROMs tests were again carried out on samples taken from all patients. All the subjects involved in our study have reached the measurements at 30 and 90 days.

A statistical package software (Statistical Package for Social Sciences SPSS® version 20.0) was used for the analysis. All the data were expressed as mean value \pm SD. Statistical significance between comparable data was calculated by means of Student's t-test and ANOVA Test. Values less than 0.05 were considered as statistically significant.

\section{Results}

Our results showed that patients affected by chronic apical periodontitis presented higher levels of systemic oxidative stress than the general population. On recruitment, the mean value of the d-ROMs test was $458 \pm 36$, while the mean value of the BAP test was $1790 \pm 64$ in patients inside the "Group 1". Conversely, the values of patients in "Group 2" were normal, 
with values of $261 \pm 10$ for the d-ROMs test and $2227 \pm$ 55 for the BAP test.

After endodontic treatment of chronic apical periodontitis, the levels of oxidative stress were generally found to have progressively returned to normal in the "Group 1" subjects. Indeed, the first follow-up visit at 30 days after treatment $\left(\mathrm{T}_{2}\right)$ revealed an average reduction in the value of the d-ROM test of $25 \%$ and an average increase in the value of the BAP test of $11 \%$. Conversely, the values of the subjects in "Group 2 " remained nearly constant with a $\mathrm{T}_{0}-\mathrm{T}_{2} \mathrm{~d}$-ROMs test variation of $4 \%$ and a $\mathrm{T}_{0}-\mathrm{T}_{2}$ BAP test variation of $5 \%$.

The second follow-up visit at 90 days $\left(\mathrm{T}_{3}\right)$ revealed a further average increase in the BAP test value of $20 \%$ and a further average reduction in the d-ROM test value of $26 \%$, whereas the values of the subjects in "Group 2" remained nearly constant with a $\mathrm{T}_{0}-\mathrm{T}_{3}$ d-ROMs test variation of $10 \%$ and a $\mathrm{T}_{0}-\mathrm{T}_{3}$ BAP test variation of $4 \%$. (Table 1-2).

The purpose of the statistical analysis between the values observed in Group 1 and those observed in Group 2 is aimed at assess whether the difference of the absolute values between a group "Test" and a group "Control" is also statistically relevant, although the parameters on the basis of which both groups must be analyzed are already known, thanks to the standardized method that we have used; in fact, the normal values of $d-R O M s$ test range between 250 and 300 CARR U, instead, the normal value of a BAP test is $>2200 \mathrm{uM}$.

Table I. Analysis of chronic apical periodontitis group (Group I).

\begin{tabular}{|l|l|l|l|l|l|l|l|l|l|l|}
\hline Group 1 & \multicolumn{9}{|c|}{ BAP Test $(\boldsymbol{\mu M} / \mathbf{L})$} & \multicolumn{5}{|c|}{ d-ROMs Test (U CARR) } \\
\hline & $\mathrm{T}_{0}$ & $\mathrm{~T}_{2}$ & $\mathrm{~T}_{3}$ & $\begin{array}{l}\mathrm{T}_{0}-\mathrm{T}_{2} \\
\text { variation }\end{array}$ & $\begin{array}{l}\mathrm{T}_{0}-\mathrm{T}_{3} \\
\text { variation }\end{array}$ & $\mathrm{T} 0$ & $\mathrm{~T}_{2}$ & $\mathrm{~T}_{3}$ & $\begin{array}{l}\mathrm{T}_{0}-\mathrm{T}_{2} \\
\text { variation }\end{array}$ & $\begin{array}{l}\mathrm{T}_{0}-\mathrm{T}_{3} \\
\text { variation }\end{array}$ \\
\hline mean & $1790 \pm 64$ & $1847 \pm 65$ & $2365 \pm 110$ & $11 \%$ & $20 \%$ & $458 \pm 36$ & $311 \pm 30$ & $274 \pm 14$ & $25 \%$ & $26 \%$ \\
\hline F & n.d. & n.d. & n.d. & 144,0542 & 679,6816 & n.d. & n.d. & n.d. & 275,2317 & 555,9293 \\
\hline Crit. F & n.d. & n.d. & n.d. & 3,88745 & 3,88745 & n.d. & n.d. & n.d. & 3,88745 & 3,88745 \\
\hline
\end{tabular}

Table 2. Analysis of healthy group (Group 2).

\begin{tabular}{|l|l|l|l|l|l|l|l|l|l|l|}
\hline Group 2 & \multicolumn{4}{|c|}{ BAP Test $(\boldsymbol{\mu M} / \mathbf{L})$} & \multicolumn{4}{c|}{ d-ROMs Test (U CARR) } \\
\hline & $\mathrm{T}_{0}$ & $\mathrm{~T}_{2}$ & $\mathrm{~T}_{3}$ & $\begin{array}{l}\mathrm{T}_{0}-\mathrm{T}_{2} \\
\text { variation }\end{array}$ & $\begin{array}{l}\mathrm{T}_{0}-\mathrm{T}_{3} \\
\text { variation }\end{array}$ & $\mathrm{T} 0$ & $\mathrm{~T}_{2}$ & $\begin{array}{l}\mathrm{T}_{3} \\
\mathrm{~T}_{0}-\mathrm{T}_{2} \\
\text { variation }\end{array}$ & $\begin{array}{l}\mathrm{T}_{0}-\mathrm{T}_{3} \\
\text { variation }\end{array}$ \\
\hline mean & $2227 \pm 55$ & $2269 \pm 73$ & $2258 \pm 66$ & $5 \%$ & $4 \%$ & $261 \pm 10$ & $273 \pm 20$ & $275 \pm 20$ & $4 \%$ & $10 \%$ \\
\hline $\mathbf{F}$ & n.d. & n.d. & n.d. & 4,12 & 1,03 & n.d. & n.d. & n.d. & 0,19 & 0,00 \\
\hline Crit. F & n.d. & n.d. & n.d. & 3,89 & 3,89 & n.d. & n.d. & n.d. & 3,89 & 3,89 \\
\hline
\end{tabular}

As is shown in Table 3, the data of BAP at $\mathrm{T}_{0}$ and at $T_{2}$ are statistically significant $(P<0.05)$, however, other data, compared with the Student 's t-test, show a lack of statistical significance: this is not surprising, in fact the data measured at time $\mathrm{T}_{0}$ express parameters altered, due to the influence of the CAP, with respect to Group 2, where instead the parameters are more in conformity to the standards of normality; in addition, a significance is present in the comparison between the values measured at time $T_{2}$ in the two groups, and this is explained by the fact that the chronic apical periodontitis, even if it is treated with an endodontic therapy, it leads to having in the following days the typical reparative processes which lead to the activation of biochemical processes that could justify such a difference in the values recorded between the two groups. The results, therefore, are indicative of an effective influence of the endodontic pathology chronic on the levels of systemic oxidative stress in untreated patients and in those treated, up to 30 days after endodontic therapy.
Table 3. Comparisons between CAP group (Group I) and Control group (Group 2).

\begin{tabular}{|l|l|l|l|}
\hline Group 1 vs. Group 2 & Timing & Significance & P value \\
\hline \multirow{4}{*}{ BAP Test $(\mu \mathrm{M} / \mathrm{L})$} & $\mathrm{T}_{0}$ & Yes & 0,035 \\
\cline { 2 - 4 } & $\mathrm{T}_{2}$ & Yes & 0,048 \\
\cline { 2 - 4 } & $\mathrm{T}_{3}$ & No & 0.492 \\
\hline \multirow{2}{*}{ d-ROMs Test $(\mathrm{U}$} & $\mathrm{T}_{0}$ & No & 0.157 \\
\cline { 2 - 4 } & $\mathrm{T}_{2}$ & No & 0.112 \\
\cline { 2 - 4 } & $\mathrm{T}_{3}$ & No & 0.971 \\
\hline
\end{tabular}

$\mathrm{T} 0=$ before endodontic treatment;

$\mathrm{T} 2=30$ days after endodontic treatment;

$\mathrm{T} 3=90$ days after endodontic treatment.

Biological antioxidant test (BAP) test n.v. $>2200 \mu \mathrm{M}$ of reduced iron for L plasma examined.

Determination of reactive oxygen metabolites (d-ROMs) test n.v. 250- 300 U CARR. $\mathrm{F}$ is the variation between mean values. The higher is $\mathrm{F}$, the more significant is the difference between mean values. Critical $F$ value is statistically significant if $p<0,05$; therefore, if $\mathrm{F}$ is higher than critical $\mathrm{F}$, the difference is statistically significant. In other words, critical FR value tells us that $\mathrm{F}$ needs to be higher than critical $\mathrm{F}$ to be significant at the $0,05 \%$. 


\section{Discussion}

Systemic oxidative stress has been correlated with several pathological or physiopathological conditions such as type 2 diabetes mellitus, aging, cardiovascular diseases, cancer, pulmonary diseases, and hepatopathies. For instance, in type 2 diabetes mellitus, ROS have been shown to be able to impair the action of insulin, thereby contributing to increased glycemia. Compounding this effect, insulin resistance and hyperglycemia have also been shown to lead to both an increased production of ROS and a reduction in antioxidant defenses. ROS have also been found to be important in the pathogenesis of the complications of diabetes, such as neuropathy, cardiovascular diseases, and the impairment of microbicidal activity 3,4 . In addition to a role in the pathogenesis of diabetes, ROS production has also been correlated with factors, such as overweight, caloric excess, and inadequate physical activity, which contribute to aging. Indeed, these factors increase mitochondrial free radical production and reduce the efficiency of antioxidant systems, which in turn increases the morbidity of cardiovascular diseases and neoplasias 5, 6. Not surprisingly, ROS also play a role in cardiovascular diseases. In most cases, these diseases are caused by atherosclerosis, the pathogenesis of which is mainly related to the oxidation of low density lipoproteins (LDL) by free radicals. ROS also cause ischemia-reperfusion injury through the activation of xanthine oxidase ${ }^{7-11}$. In cancer, ROS play a crucial role, especially in tumors caused by ionizing radiations, where they cause photolysis of water and produce the hydroxyl radical. As the hydroxyl radical is able to break DNA filaments or oxidize bases, the ensuing mutagenic effect can promote neoplastic transformation 12-14-20. Over the last few years, many studies have also highlighted the role of ROS in the pathogenesis of several airway diseases, such as the effects of smoking, chronic injuries (pulmonary emphysema, interstitial fibrosis), and acute manifestations (adult respiratory distress syndrome) 15,16. Finally, ROS have also been implicated in hepatopathies. In particular, enzymatic systems responsible for the metabolism of ethanol and xenobiotics are present in the liver, while microsomes are directly responsible for the production of ROS. The imbalance between pro-oxidant and anti-oxidant status is considered to be responsible for the cellular damage that can be observed in alcoholic or drug induced hepatopathies 3,4 .

Despite the association of ROS with such a wide variety of pathologies, thus far, few studies have investigated the relationship between diseases of endodontic origin and oxidative stress. The present study has therefore examined the relationship between chronic apical periodontitis and systemic oxi- dative stress. Our results demonstrate that subjects affected by chronic apical periodontitis are exposed to a condition of higher general oxidative stress compared to general population, and this is extremely dangerous for the general health: the condition of oxidative stress shows its primary action near the site where it is present the pathogenic noxa, in fact, the studies conducted so far have evaluated exclusively the changes found in crevicular fluid of patients investigated; however, an influence of chronic endodontic disease on the levels of systemic oxidative stress can be a significant contributing cause in the pathogenesis of some serious systemic diseases, going on increasing thus the risk factor of patients with CAP, compared to the general population. Moreover, an additional potential problem, which could arise from the increase in systemic oxidative stress in patients with CAP, is the acceleration of clinical onset of certain diseases that would otherwise have a sub-clinical course longer, with less damage to the patient and, therefore, also with minor impact on public health spending.

Minczykowski et al. (2001) investigate changes in superoxide anion and hydrogen peroxide production by polymorphonuclear neutrophils (PMN) in patients with chronic periapical granuloma before and after surgical treatment; if, on the one hand, our study shows that the reduction of oxidative stress, evidenced by the change of the values obtained by $\mathrm{d}$-ROMs and BAP tests, is clearly evident 30 days after endodontic treatment, confirming our observations, and Minczykowski collaborators have observed that already 14 days after the removing the cause of inflammation, which in this case was done by the extraction of the carious tooth, one could observe that O2-production by unstimulated cells was higher than the controls and lower in comparison to PMNs taken before treatment, in addition, $\mathrm{H} 2 \mathrm{O} 2$ production was not higher when compared to controls and significantly lower in comparison with PMNs taken before tooth extraction ${ }^{20}$. The presence of chronic apical periodontitis leads, if untreated, damages that cause the loss of periodontal supporting tissue ${ }^{21}$ : this destructive activity is also expressed in the absence of symptomatology, and in support of this, it is possible to highlight the presence of inflammatory mediators, metalloproteinases in gingival crevicular fluid of teeth affected by with CAP.

In a recent study, Dezerega et al. (2012) have studied the activity of metalloproteinase 2 and metalloproteinase 9, which have shown an increased activity during the state of periapical inflammation; moreover, the gingival crevicular fluid showed lower levels of total antioxidant status in diseased teeth at 
baseline compared to endodontically-treated groups ${ }^{22}$.

Despite in literature there are really few studies who have investigated about this topic, the majority of these studies have achieved out results in line with the present study, therefore, the presence of oxidant imbalance in the patients affected by chronic apical periodontitis, whereas it's quite clear as the endodontic treatment restores antioxidant status to its normal levels. Our study has, therefore, confirmed an association between CAP and oxidative stress, and it has also demonstrated, with biological data, that a cohort of patients with CAP undergoes a significant increment of systemic oxidative stress: in the light of the many studies in which has been shown that the oxidative stress plays a role in the pathogenesis of many diseases, and in light of the fact that we have shown that even a simple CAP is able to elevate the levels of systemic oxidative stress, the new question to be investigated is: how much the co-presence of endodontic diseases can increase the risk parameters of an healthy person to develop systemic diseases? Moreover, the presence of diseases correlated to the increased levels of oxidative stress, might have a more aggressive development, as a result of untreated or undetected CAP? Surely, this study induces new questions that lead to reflect about the role of a proper diagnosis carried out by the dentist, which becomes an important opportunity of prevention.

Further studies are needed to confirm these suggestive hypotheses, as well as it's already unclear the biochemical pathway which clearly explains how this physiological mechanism works.

In conclusion, our study, as well as other studies in the literature, underlines the correspondence between CAP and oxidative stress, so it is important to analyze the values of this parameter to limit the damage that the periodontal tissues may undergo, even in the absence of symptoms. Moreover, one can infer from these findings that through proper endodontic therapy, a good oxidative balance can be restored, thereby avoiding the risk of having a chronic focus, able to cause damages with potentially generalized consequences.

\section{Acknowledgments}

We thank the Department of Endodontics, Calabrodental Clinic in Crotone, for the cooperation. We thank dr. Francesco Paduano for his help in the statistical analysis.

\section{Competing Interests}

The authors have declared that no competing interest exists.

\section{References}

1. Ferreiro-Vera C, Ribeiro JP, Mata-Granados JM, Priego-Capote F, Luque de Castro MD. Standard operation protocol for analysis of lipid hydroperoxides in human serum using a fully automated method based on solid-phase extraction and liquid chromatography-mass spectrometry in selected reaction monitoring. J Chromatogr A. 2011; 1218(38):6720-6.

2. Gerardi GM, Usberti M, Martini G, et al. Plasma total antioxidant capacity in hemodialyzed patients and its relationship to other biomarkers of oxidative stress and lipid peroxidation. Clin Chem Lab Med. 2002; 40:104-110.

3. Pasquini A, Luchetti E, Marchetti V, Cardini G, Iorio EL. Analytical performances of d-ROMs test and BAP test in canine plasma. Definition of the normal range in healthy Labrador dogs. Vet Res Commun. 2008; 32:137-43.

4. Elbim C, Lizard G. Flow cytometric investigation of neutrophil oxidative burst and apoptosis in physiological and pathological situations. Cytometry A. 2009;75(6):475-81.

5. Spiteller G. Is lipid peroxidation of polyunsaturated acids the only source of free radicals that induce aging and age-related diseases? Rejuvenation Res. 2010;13(1):91-103.

6. Clark TA, Lee HP, Rolston RK, Zhu X, Marlatt MW, Castellani RJ, Nunomura A, Casadesus G, Smith MA, Lee HG, Perry G. Oxidative Stress and its Implications for Future Treatments and Management of Alzheimer Disease. Int J Biomed Sci. 2010; 6(3):225-227.

7. Shao D, Oka SI, Brady CD, Haendeler J, Eaton P, Sadoshima J. Redox modification of cell signaling in the cardiovascular system. J Mol Cell Cardiol. 2011 Sep 17.

8. Digiesi V, Oliviero C, Gianno V, et al. Reactive metabolites of oxygen, lipid peroxidation, total antioxidant capacity and vitamin $\mathrm{E}$ in essential arterial hypertension. Clin Ter. 1997; 148:515-519.

9. Incandela L, Belcaro G, Cesarone MR, et al. Oxygen-free radical decrease in hypertensive patients treated with lercanidipine. Int Angiol. 2001; 20:136-40.

10. Li R, Jen N, Yu F, Hsiai TK. Assessing mitochondrial redox status by flow cytometric methods: vascular response to fluid shear stress. Curr Protoc $\mathrm{Cy}-$ tom. 2011;

11. Cesarone MR, Incandela L, DeSanctis MT, Belcaro G, Dugall M, Acerbi G. Variations in plasma free radicals in patients with venous hypertension with HR: a clinical, prospective, placebo-controlled, randomized trial. J Cardiovasc Pharmacol Ther. 2002; 7:25-8

12. Iorio EL, Quagliuolo L, Carratelli M. The d-ROMs test: a method to monitor oxidative stress in vascular diseases. Minerva Cardioangiol. 2002; 50:143-4.

13. Cortelezzi A, Fracchiolla NS, Bamonti-Catena F, Motta M, Cighetti G, Carrabba M, Cavalca V, Maiolo AT, Lambertenghi Deliliers G. Hyperhomocysteinemia in myelodysplastic syndromes: specific association with autoimmunity and cardiovascular disease. Leuk Lymphoma. 2001; 41(1-2):147-50.

14. Ghoti H, Fibach E, Merkel D, Perez-Avraham G, Grisariu S, Rachmilewitz EA. Changes in parameters of oxidative stress and free iron biomarkers during treatment with deferasirox in iron-overloaded patients with myelodysplastic syndromes. Haematologica. 2010; 95(8):1433-4.

15. La Torre F, Orlando A, Silipigni A, Giacobello T, Pergolizzi S, Aragona M. Increase of oxygen free radicals and their derivatives in chemo- and radiation treated neoplasm patients. Minerva Med. 1996; 86:1-4.

16. Kamboj SS, Sandhir R. Protective effect of N-acetylcysteine supplementation on mitochondrial oxidative stress and mitochondrial enzymes in cerebral cortex of streptozotocin-treated diabetic rats. Mitochondrion. 2011;11(1):214-22.

17. Giuliana G, Ammatuna P, Pizzo G, Capone F, D'Angelo M. Occurrence of invading bacteria in radicular dentin of periodontally diseased teeth: microbiological findings. J Clin Perio 1997; 7(24): 478-485.

18. Steeves $\mathrm{CH}$, Potrykus J, Barnett DA, Bearne SL. Oxidative stress response in the opportunistic oral pathogen Fusobacterium nucleatum. Proteomics. 2011;11(10):2027-37.

19. Zaragoza C, Lopez-Rivera E, Garcia-Rama C, Saura M, Martinez-Ruiz A, Lizarbe TR, Martin-de-Lara F, Lamas S. Cbfa-1 mediates nitric oxide regulation of MMP-13 in osteoblasts. J Cell Sci 2006;119:1896-1902.

20. Minczykowski A, Woszczyk M, Szczepanik A, Lewandowski L, Wysocki H. Hydrogen peroxide and superoxide anion production by polymorphonuclear neutrophils in patients with chronic periapical granuloma, before and after surgical treatment. Clin Oral Investig. 2001; 5(1):6-10.

21. Gutmann JL, Baumgartner JC, Gluskin AH, Hartwell GR, Walton RE. Identify and define all diagnostic terms for periapical/periradicular health and disease states. J Endod 2009, 35:1658-1674.

22. Dezerega A, Madrid S, Mundi V, Valenzuela MA, Garrido M, Paredes R, García-Sesnich J, Ortega AV, Gamonal J, Hernández M. Pro-oxidant status and matrix metalloproteinases in apical lesions and gingival crevicular fluid as potential biomarkers for asymptomatic apical periodontitis and endodontic treatment response. J Inflamm (Lond). 2012 Mar 21; 9(1):8. 